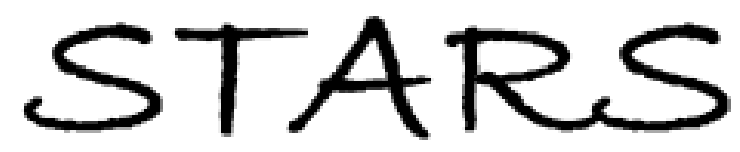

University of Central Florida

STARS

$1-1-2004$

\title{
Fast-response and scattering-free polymer network liquid crystals for infrared light modulators
}

\author{
Yun-Hsing Fan \\ University of Central Florida \\ Yi-Hsin Lin \\ University of Central Florida \\ Hongwen Ren \\ University of Central Florida \\ Sebastian Gauza \\ University of Central Florida \\ Shin-Tson Wu \\ University of Central Florida \\ Find similar works at: https://stars.library.ucf.edu/facultybib2000 \\ University of Central Florida Libraries http://library.ucf.edu
}

This Article is brought to you for free and open access by the Faculty Bibliography at STARS. It has been accepted for inclusion in Faculty Bibliography 2000s by an authorized administrator of STARS. For more information, please contactSTARS@ucf.edu.

\section{Recommended Citation}

Fan, Yun-Hsing; Lin, Yi-Hsin; Ren, Hongwen; Gauza, Sebastian; and Wu, Shin-Tson, "Fast-response and scattering-free polymer network liquid crystals for infrared light modulators" (2004). Faculty Bibliography 2000s. 4343.

https://stars.library.ucf.edu/facultybib2000/4343

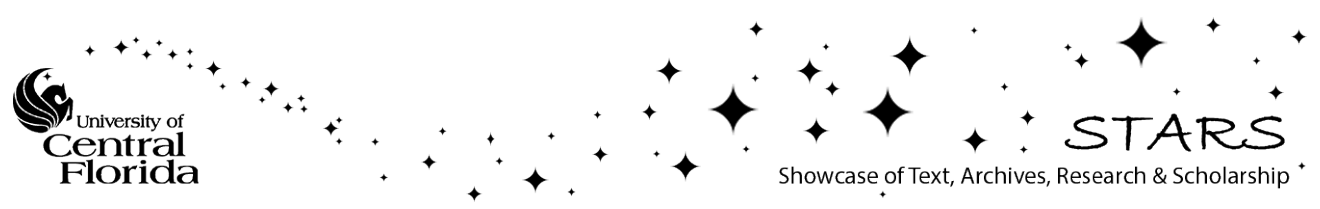




\section{Fast-response and scattering-free polymer network liquid crystals for infrared light modulators}

Cite as: Appl. Phys. Lett. 84, 1233 (2004); https://doi.org/10.1063/1.1649816

Submitted: 30 October 2003 . Accepted: 19 December 2003 . Published Online: 18 February 2004

Yun-Hsing Fan, Yi-Hsin Lin, Hongwen Ren, Sebastian Gauza, and Shin-Tson Wu

\section{ARTICLES YOU MAY BE INTERESTED IN}

Fast-switching initially-transparent liquid crystal light shutter with crossed patterned electrodes

AIP Advances 5, 047118 (2015); https://doi.org/10.1063/1.4918277

Cholesteric liquid crystal/polymer dispersion for haze-free light shutters Applied Physics Letters 60, 3102 (1992); https://doi.org/10.1063/1.106765

Network morphology of polymer stabilized liquid crystals

Applied Physics Letters 71, 2454 (1997); https://doi.org/10.1063/1.120087

\section{Applied Physics Letters}

Mid-IR and THz frequency combs special collection

\section{Read Now!}




\title{
Fast-response and scattering-free polymer network liquid crystals for infrared light modulators
}

\author{
Yun-Hsing Fan, Yi-Hsin Lin, Hongwen Ren, Sebastian Gauza, and Shin-Tson Wu ${ }^{\text {a) }}$ \\ School of Optics/CREOL, University of Central Florida, Orlando, Florida 32816
}

(Received 30 October 2003; accepted 19 December 2003)

\begin{abstract}
A fast-response and scattering-free homogeneously aligned polymer network liquid crystal (PNLC) light modulator is demonstrated at $\lambda=1.55 \mu \mathrm{m}$ wavelength. Light scattering in the near-infrared region is suppressed by optimizing the polymer concentration such that the network domain sizes are smaller than the wavelength. The strong polymer network anchoring assists LC to relax back quickly as the electric field is removed. As a result, the PNLC response time is $\sim 250 \times$ faster than that of the E44 LC mixture except that the threshold voltage is increased by $\sim 25 \times$. (C) 2004 American Institute of Physics. [DOI: 10.1063/1.1649816]
\end{abstract}

Liquid crystal/polymer composites could appear in network or droplet form depending on what polymer and concentration is employed. In the low concentration regime (1-2 wt $\%)^{1,2}$ the polymer field improves the response time, however, the associated light scattering is quite strong in the visible region. As the polymer concentration increases to $\sim 3 \%-8 \%$, the polymer-stabilized liquid crystal (PSLC; also known as gel) is formed in a homogeneous or homeotropic cell. ${ }^{3,4}$ These PSLC cells exhibit an anisotropic light scattering behavior and has potential for reflective display and telecom applications. ${ }^{5,6}$ As the polymer concentration increases to $\sim 30 \%-40 \%$, the polymer-dispersed liquid crystal (PDLC) is formed. ${ }^{7,8}$ No surface alignment in the PDLC cell is necessary. These micron-sized droplets scatter visible light and this scattering is independent of polarization. In the high polymer concentration regime $(60 \%-70 \%)$, the nanoscale holographic PDLC (HPDLC) droplets are formed. ${ }^{9,10}$ Since the droplet size is smaller than the visible wavelength, HPDLC cell does not scatter light and its response time is fast $(\sim 200 \mu \mathrm{s})$. However, to reorient these LC droplets would require a relatively high electric field $(\sim 15-20$ $\left.\mathrm{V}_{\text {rms }} / \mu \mathrm{m}\right)$. Recently, a stressed LC cell comprised of 14 wt $\%$ Nolan-65 polymer was demonstrated to exhibit a fast response time. ${ }^{11}$ In this approach, no surface alignment is required. However, a delicate shearing process is needed in order to suppress light scattering. The operating voltage for a $20 \mu \mathrm{m}$ E7 cell is $\sim 135 \mathrm{~V}_{\mathrm{rms}}$.

In this letter, we report a fast-response homogeneously aligned polymer network liquid crystal (PNLC) cell comprised of $\sim 10 \%$ polymers. A unique feature of this PNLC is that light scattering at $\lambda=1.55 \mu \mathrm{m}$ is suppressed completely. The measured response time is less than $2 \mathrm{~ms}$ for a $2 \pi$ phase change at $\lambda=1.55 \mu \mathrm{m}$ and $T \sim 21^{\circ} \mathrm{C}$. The tradeoff of the PNLC cell is its high operating voltage. To reduce operating voltage, a higher birefringence and larger dielectric anisotropic LC can be considered.

To fabricate the PNLC cells, we first mixed a few percent of photocurable monomer to a nematic LC host. The $\mathrm{LC} /$ monomer cell was then cured using an UV lamp. Two

a)Electronic mail: swu@mail.ucf.edu types of diacrylate monomers: bisphenol-A-dimethacrylate (M1) and RM-82 (Merck) were used. The melting point of these two monomers is 74.6 and $82.4{ }^{\circ} \mathrm{C}$, respectively. Due to the high heat fusion enthalpy, each monomer's solubility is limited to 7-8 wt \%. Under these conditions, the cured polymer network domain size is still too large, which results in $\sim 15 \%$ light scattering loss at $\lambda=1.55 \mu \mathrm{m}$. In order to eliminate light scattering, we need to reduce the domain size further by increasing the monomer concentration. Therefore, we mixed M1 with RM-82. For the LC mixture (Merck E44) we studied, the best monomer concentration is $6 \mathrm{wt} \% \mathrm{M} 1$ and 4 wt $\%$ RM-82. Unless otherwise mentioned throughout this letter, our LC/polymer contains 90\% E44, 6\% M1, and 4\% RM-82.

For laser beam steering at $\lambda=1.55 \mu \mathrm{m}$, both reflective and transmissive phase modulators can be used. ${ }^{12}$ To generate diffractive phase gratings, a $2 \pi$ phase change between adjacent grating elements is required. The reflective and transmissive PNLC cells we prepared have, respectively, 7.7 and $12.5 \mu \mathrm{m}$ gap. The inner surfaces of the indium-tin-oxide glass substrates were over-coated with a thin polyimide layer and buffed in antiparallel directions for achieving homogeneous alignment. The filled cell was then exposed to UV light for curing the polymer networks. To eliminate light scattering, we need to control the domain sizes to be smaller than the wavelength. A weak UV intensity would result in coarser polymer networks which, in turn, lead to larger domain sizes. Thus, we exposed the LC/monomer mixture using a high UV intensity $\left(I \sim 45 \mathrm{~mW} / \mathrm{cm}^{2}\right)$. The exposure time is $30 \mathrm{~min}$. The resultant polymer networks align, on average, parallel to the substrate surfaces due to the strong surface anchoring energy. Thus, the PNLC cell is highly transparent in the voltage-off state. In a voltage-on state, the polymer networks exert a torque to resist the LC molecules from being reoriented by the electric field. As a result, the threshold voltage is increased significantly. Once the electric field is removed, the strong polymer field assists LC to relax back quickly.

Figure 1 depicts the experimental setup for studying the electro-optic effects of the reflective and transmissive PNLC cells. The incoming diode laser beam $(\lambda=1.55 \mu \mathrm{m})$ is reflected or transmitted by the polarizing beam splitter to the 


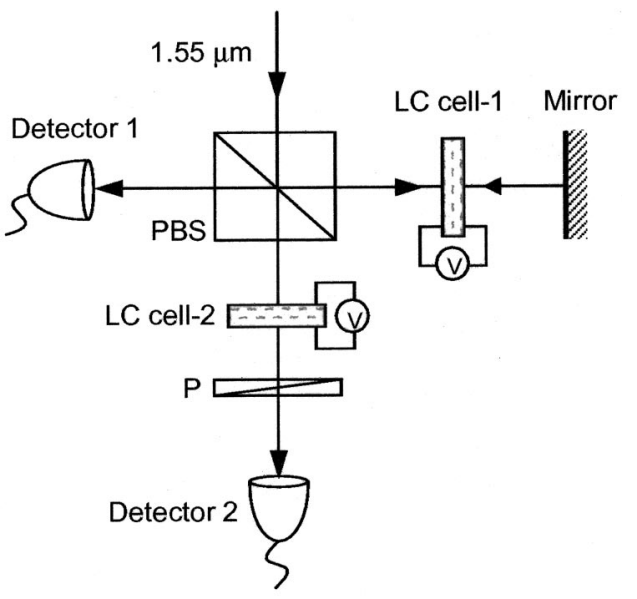

FIG. 1. Experimental setup for measuring the electro-optic properties of the reflective and transmissive LC and PNLC cells. The rubbing direction of the cell is at $45^{\circ}$ to the polarization axis of the incident light. The LC cell- 1 and detector 1 are for reflective mode and LC cell-2 and detector 2 are for transmissive mode. $\mathrm{PBS}=$ polarizing beam splitter. $\mathrm{P}=$ polarizer. $\lambda=1.55$ $\mu \mathrm{m}$.

PNLC cell. The rubbing direction of the LC cell is oriented to be $45^{\circ}$ to the polarization axis of the incident beam. For the reflective mode, ideally the reflector should be imbedded in the inner side of the rear substrate in order to eliminate multiple surface reflections. For feasibility studies, we placed a dielectric mirror behind the LC cell to serve as a reflector. For the transmissive mode, the analyzer is crossed to the polarizer. The voltage-dependent reflectance or transmittance
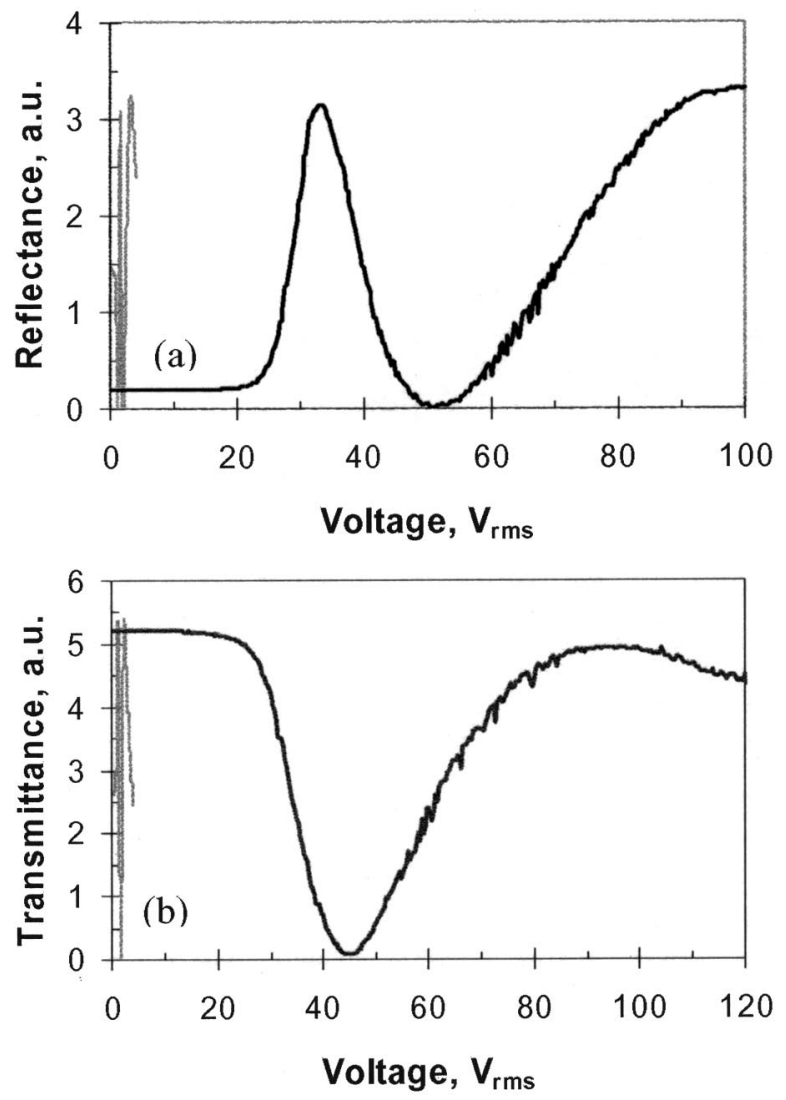

FIG. 2. Voltage-dependent reflectance (a) and transmittance (b) of E44 (gray lines) and PNLC (dark lines) cells. The cell gap for the reflective and transmissive cells is 7.7 and $12.5 \mu \mathrm{m}$, respectively. $\lambda=1.55 \mu \mathrm{m}$ and $T=21^{\circ} \mathrm{C}$.
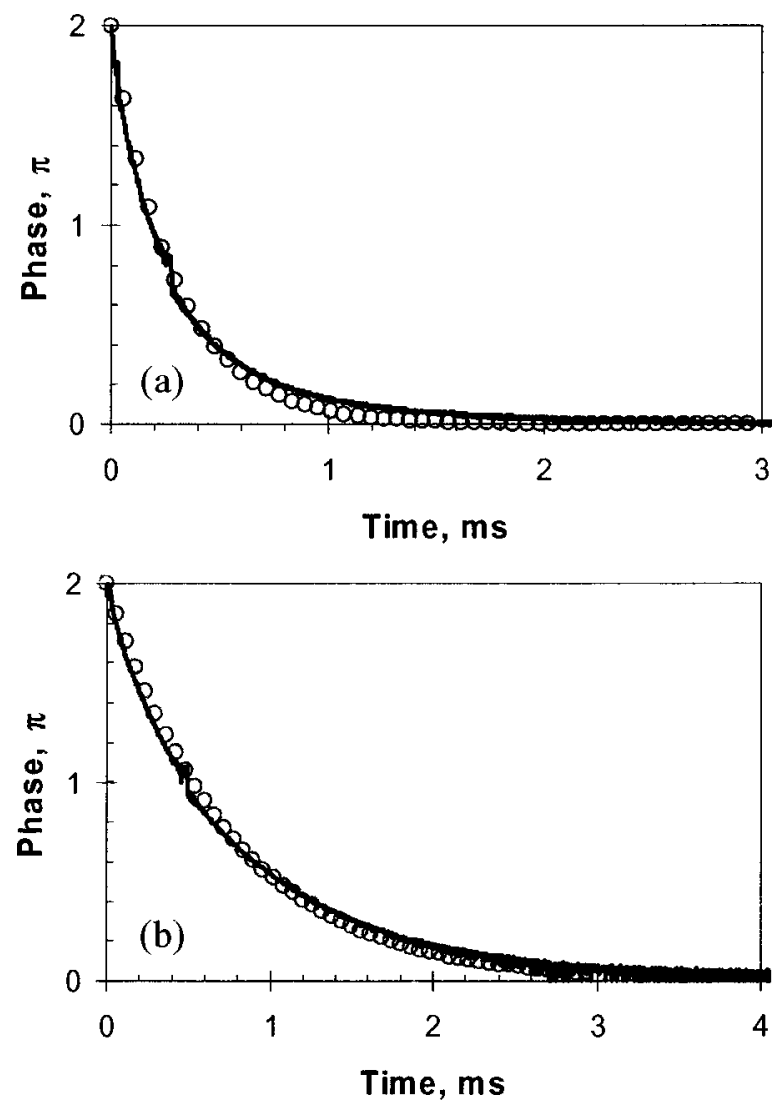

FIG. 3. Measured phase decay time of the reflective (a) and transmissive (b) PNLC cells. Solid lines are experimental data and open circles are fitting results using $\delta(t)=2 \pi \exp (-t / \tau)$. In (a), $\tau=296 \mu \mathrm{s}$ and in (b) $\tau=755 \mu \mathrm{s}$. $T=21^{\circ} \mathrm{C}$ and $\lambda=1.55 \mu \mathrm{m}$.

of the cells is recorded by the LABVIEW system and data are analyzed by a computer.

Figures 2(a) and 2(b) plot the voltage-dependent reflectance and transmittance of the corresponding 7.7 and 12.5 $\mu \mathrm{m}$ E44 PNLC cells. The total phase retardation of the reflective and transmissive PNLC cells is measured to be $\delta=3.84 \pi$ and $3.0 \pi$, respectively. However, the last $\pi$ phase change would require a very large voltage swing. For the interest of keeping operating voltage low, we operated the LC modulator from $\delta=2 \pi$ (where $\left.V=V_{2 \pi}\right)$ to $0(V=0)$. Also included in Figs. 2(a) and 2(b) (gray lines) for comparisons are the 7.7 and $12.5 \mu \mathrm{m}$ E44 cells without polymer. The threshold voltage of the E44 cells is $\sim 0.9 \mathrm{~V}_{\text {rms }}$. From the measured total phase retardation, the birefringence of E44 at $\lambda=1.55 \mu \mathrm{m}$ is $\Delta n \sim 0.21$ at $T \sim 21^{\circ} \mathrm{C}$. On the other hand, the $\Delta n$ of the PNLC cell was measured to be $\Delta n \sim 0.19$. The lower $\Delta n$ originates from the imbedded $10 \%$ polymers. This result indicates that the polymer networks do not disturb LC alignment noticeably. The threshold voltage of the 7.7 and $12.5 \mu \mathrm{m}$ PNLC cells is increased to $V_{\mathrm{th}} \sim 24$ and $\sim 28 \mathrm{~V}_{\mathrm{rms}}$, respectively. The increased threshold voltage implies that the LC molecules are tightly anchored by the polymer networks. In Figs. 2(a) and 2(b), the peak transmittance of the PNLC cells is similar to that of the pure E44 cells. This indicates that the light scattering is eliminated. We also measured the wavelength-dependent transmittance of the $12.5 \mu \mathrm{m}$ cell. Scattering is strong in the visible region but becomes negligible as $\lambda>1 \mu \mathrm{m}$.

Figures 3(a) and 3(b) show the measured decay time 

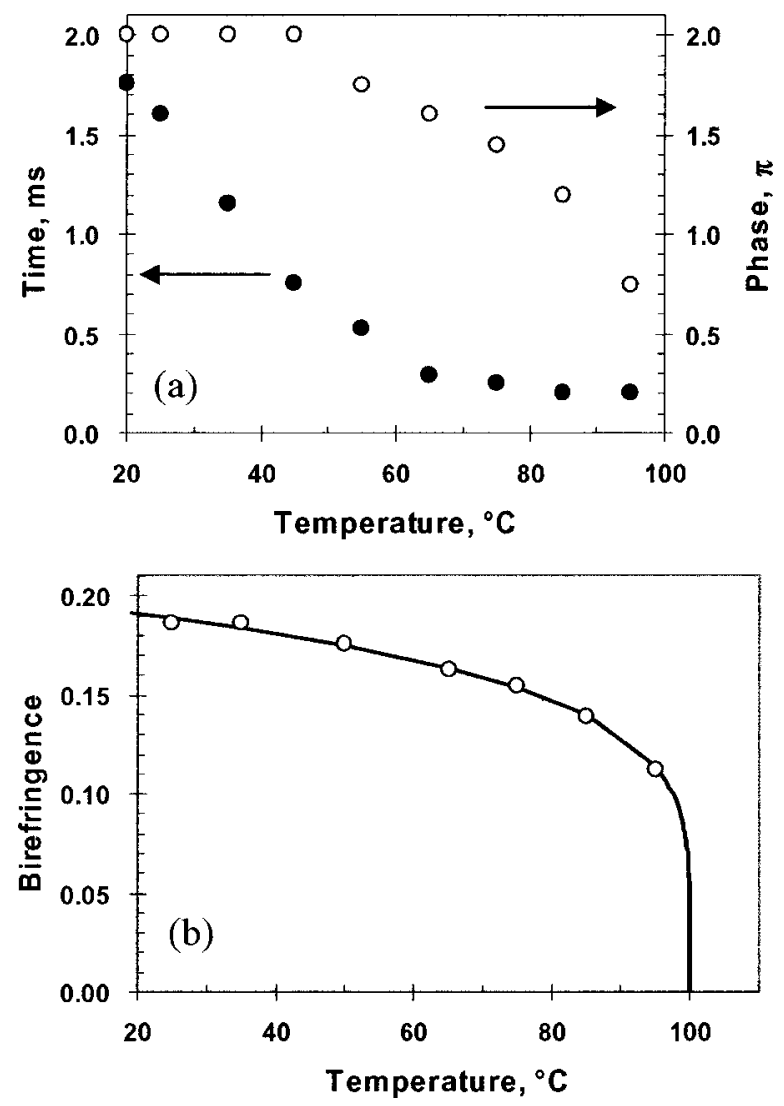

FIG. 4. Temperature-dependent LC response time (a) and birefringence (b) of the E44 transmissive PNLC cell. $d=12.5 \mu \mathrm{m}$. Closed circles are the measured response time and open circles are the corresponding phase change.

(solid lines) of the reflective and transmissive PNLC cells. The $7.7 \mu \mathrm{m}$ reflective and $12.5 \mu \mathrm{m}$ transmissive cells were initially biased at $V_{2 \pi}=58$ and $90 \mathrm{~V}_{\text {rms }}$, respectively. At $t=0$, the voltage was removed spontaneously and optical signal was recorded by a photodiode detector. The open circles are fittings with the exponential phase decay function: $\delta(t)$ $=2 \pi \exp (-t / \tau){ }^{13}$ Good agreement is obtained. The time constant (at 1/e) for the $7.7 \mu \mathrm{m}$ reflective cell and $12.5 \mu \mathrm{m}$ transmissive cell is $\tau=296$ and $755 \mu$ s, respectively. If we count the phase decay time from $100 \% \rightarrow 10 \%$, they are $\sim 0.7$ and $1.8 \mathrm{~ms}$, respectively. As compared to the pure E44 cell, the response time is improved by $\sim 250 \times$. The rise time $(10 \% \rightarrow 90 \%$ phase change) was measured to be $\sim 0.5 \mathrm{~ms}$ for the $12.5 \mu \mathrm{m}$ transmissive PNLC cell operating from 0 to 90 $\mathrm{V}_{\text {rms }}$.

Figure 4(a) plots the temperature-dependent decay time (closed circles) and the corresponding phase (open circles) of the $12.5 \mu \mathrm{m}$ transmissive PNLC cell. As the temperature increases, the operating voltage, birefringence, and response time all decrease. At $T=65^{\circ} \mathrm{C}$, the decay time for the transmissive PNLC cell is reduced to $\sim 300 \mu \mathrm{s}$, although the phase change studied is reduced to $1.6 \pi$. Figure 4(b) shows the temperature-dependent birefringence of the transmissive
E44 PNLC cell at $\lambda=1.55 \mu \mathrm{m}$. The dots represent the experimental data and solid lines are fittings with Haller's equation $\Delta n=\Delta n_{0}\left(1-T / T_{c}\right)^{\beta}$; where $\Delta n_{0}$ is the birefringence at $T=0$ and $\beta$ is a material parameter. ${ }^{14}$ From the fittings, we find $\Delta n_{0}=0.255$ and $\beta=0.187$.

To reduce the operating voltage while retaining the required $2 \pi$ phase change, we could increase the LC birefringence, dielectric anisotropy $(\Delta \epsilon)$, or cell gap. Among these three, the simplest method is to increase cell gap. In experiment, we tried a $16 \mu \mathrm{m}$ E44 PNLC cell. Indeed, its $V_{2 \pi}$ is reduced to $\sim 75 \mathrm{~V}_{\text {rms }}$ although its $V_{\text {th }}$ is increased to $\sim 30$ $\mathrm{V}_{\mathrm{rms}}$. A general drawback of thick cell approach is the increased response time. Our experimental data show that the response time of the PNLC cell is linearly proportional to the cell gap. For the $16 \mu \mathrm{m}$ cell, its decay time is increased to $\sim 3 \mathrm{~ms}$. Thus, for practical applications a proper balance between operating voltage and response time needs to be taken into consideration.

The developed PNLC can also be extended to the 3-5 and $8-12 \mu \mathrm{m}$ spectral bands. In the long wavelength region, the LC birefringence decreases ${ }^{15}$ but the light scattering becomes less significant. To obtain $2 \pi$ phase change, we need to increase the $d \Delta n$ value of the LC cell. High birefringence LC materials become crucial for these applications.

In conclusion, we have demonstrated fast-response and scattering-free reflective and transmissive PNLC phase modulators. The shortcoming is the increased voltage. To reduce operating voltage, high $\Delta n$ and large $\Delta \epsilon$ LC mixtures need to be developed. The PNLC cell also holds promise for mid- and long-infrared applications.

The authors are indebted to the financial support from DARPA STAB program through Raytheon Systems Company under Contract No. F33615-00-C-1679 and from AFOSR under Contract No. F49620-01-1-0377.

${ }^{1}$ M. Hasegawa, SID Tech. Digest 28, 699 (1997).

${ }^{2}$ M. J. Escuti, C. C. Bowley, G. P. Crawford, and S. Zumer, Appl. Phys. Lett. 75, 3264 (1999).

${ }^{3}$ R. A. M. Hikmet, J. Appl. Phys. 68, 4406 (1990).

${ }^{4}$ R. A. M. Hikmet, Mol. Cryst. Liq. Cryst. 213, 117 (1992).

${ }^{5}$ H. Ren and S. T. Wu, Appl. Phys. Lett. 81, 1432 (2002).

${ }^{6}$ F. Du and S. T. Wu, Appl. Phys. Lett. 83, 1310 (2003).

${ }^{7}$ J. L. Fergason, SID Tech. Digest 16, 68 (1985).

${ }^{8}$ J. W. Doane, N. A. Vaz, B. G. Wu, and S. Zumer, Appl. Phys. Lett. 48, 269 (1986).

${ }^{9}$ R. L. Sutherland, V. P. Tondiglia, and L. V. Natarajan, Appl. Phys. Lett. 64, 1074 (1994).

${ }^{10}$ T. J. Bunning, L. V. Natarajan, and V. P. Tondiglia, Annu. Rev. Mater. Sci. 30, 83 (2000).

${ }^{11}$ J. L. West, G. Zhang, and A. Glushchenko, SID Tech. Digest 34, 1469 (2003).

${ }^{12}$ P. F. McManamon, T. A. Dorschner, D. L. Corkum, L. Friedman, D. S. Hobbs, M. Holz, S. Liberman, H. Q. Nguyen, D. P. Resler, R. C. Sharp, and E. A. Watson, Proc. IEEE 84, 268 (1996).

${ }^{13}$ S. T. Wu and C. S. Wu, Phys. Rev. A 42, 2219 (1990).

${ }^{14}$ I. Haller, Prog. Solid State Chem. 10, 103 (1975).

${ }^{15}$ S. T. Wu, Phys. Rev. A 33, 1270 (1986). 